\title{
One-Dimensional DLR Invariant Measures are Regular
}

\author{
A. De Masi \\ Istituto di Matematica, Università degli Studi dell' Aquila, I-67100 L'Aquila, Italy
}

\begin{abstract}
A system of infinite spins in one dimension is considered. The interaction is given by a pair potential $-J_{x y} S_{x} S_{y}$, where $S_{x}, S_{y}$ are the spins at the sites $x, y \in \mathbb{Z}$ and $J_{x y}=J(|x-y|)$ where $J(|x-y|)$ decreases asymptotically in an integrable way. The self-interaction makes the system superstable. It is proven that any invariant DLR measure for this system satisfies Ruelle's superstable estimates (regularity condition).
\end{abstract}

\section{Introduction}

Gibbs random fields (DLR measures) with random variables taking on values in non-compact spaces have been extensively studied, [1-5] for applications both in statistical mechanics and lattice approximation to constructive field theory (point particles without hard-core interaction, infinite spin systems). Under reasonable hypotheses on the interaction it has been proven that there exists a set of so called regular DLR measures [see Eq. (1.2) below and Sect. 2] which have essentially the same properties as the fields in the compact case, [1-4]. The regular measures are characterized by support properties and can be explicitely constructed by taking thermodynamic limits of finite volume Gibbs measures with boundary conditions taken within an appropriate class, $[3,4]$. One is therefore induced to consider these as the physically interesting states; it is known, however, that, in general, one cannot exclude the presence of other non regular DLR measures. These have been explecitely constructed for a chain of one-dimensional nearest neighborhood interacting harmonic oscillators, [6]. To obtain them one starts with finite volume Gibbs measures and takes boundary conditions which are fastly increasing as the volume gets to infinity. The limiting measures keep memory of this process and the mean values of the harmonic oscillators are very large and not uniformly bounded. Therefore one needs "very large boundary conditions" for the limiting measure to be non regular, but this automatically leads toward a non homogenous situation. From this follows the conjecture: the translationally invariant DLR measures are regular (which is the case in the above mentioned, harmonic oscillators case). 
Different arguments suggest this same conjecture. In the compact case (the random variables take on values in a compact space) there is another way to define the translationally invariant states and this is via the "variational principle" [5]. One defines a functional on the translationally invariant probability measures which is given by $k T S(p)-E(p)$ ( $T$ is the temperature, $k$ the Boltzman constant, $S$ the entropy per unit volume and $E$ the energy density per unit volume). The probability measures for which the above functional attains its maximum value are called the equilibrium measures and in the compact case it is proven, [5] that they are the same as the DLR invariant measures. It is possible to extend the principle to the non compact case at least for "regular" probability measures but one would expect physically that it is the same principle which forces the equilibrium measures to be regular. Since one should also expect that the DLR translationally invariant measures and equilibrium measures are the same all leads again to the above conjecture. In this paper we prove the following. We consider a system of superstable one dimensional infinite spins with pair interaction of the type $-J_{x y} S_{x} S_{y}\left(x, y \in \mathbb{Z}, S_{x}, S_{y}\right.$ are the spins at $x$ and $\left.y\right)$ and free measure $\exp \left(-F\left(S_{x}\right)\right) d S_{x}$ where both $J_{x y}$ and $F\left(S_{x}\right)$ are translationally invariant. We also need a (technical) assumption of asymptotic monotonicity for $\left|J_{x y}\right|$ when $|x-y|$ diverges, while $\sum_{y \neq x}\left|J_{x y}\right|<+\infty$, to define correctly DLR measures. Under these hypotheses we prove that any translationally invariant DLR measure $v$ is regular (in the sense of Ruelle): define

$$
v\left(d S_{\Lambda}\right)=\varrho_{\Lambda}\left(S_{\Lambda}\right) d S_{\Lambda} \quad S_{\Lambda}=\left\{S_{i}, i \in \Lambda\right\}
$$

for any finite $\Lambda$, then there are constants $\gamma>0$ and $\delta \in \mathbb{R}$ both independent of $\Lambda$ such that

$$
\varrho_{\Lambda}\left(S_{\Lambda}\right) \leqq \exp \left[-\sum_{x \in \Lambda}\left(\gamma S_{x}^{2}-\delta\right)\right]
$$

To give an idea of the arguments used to prove this result we consider the nearest neighbor interactions. The first step is to obtain support properties for the translationally invariant measure $v$. By use the Birkhoff theorem we prove that with $v$-probability one in any configuration $\underline{S}$ there are infinitely many spins bounded by the same constant $C(\underline{S})$ depending possibly on the configuration $\underline{S}$. By decomposing $v$ into its extremal components (which are DLR measures with trivial algebra at infinity) we can reduce the problem to the case in which $C(\underline{S})$ does not depend on $\underline{S}$ with probability one. In this case we can use Doob's theorem and we obtain $v$ as limit of sequence of Gibbs measures at finite volume with boundary spins uniformly bounded by $C(\underline{S})$ (and therefore, also the boundary interactions is uniformly bounded). Equation (1.2) is obtained with arguments essentially contained in Ruelle's paper about superstability, $[1,2]$. Note that in this limiting process the constants $\gamma$ and $\delta$ loose memory of the constant $C(\underline{S})$ which limits the boundary spins.

In a different context other results have been obtained for one dimensional Gibbs random fields. Kesten, [7] has proven that among general Gibbs random field with countable state space (the values taken by a simple spins) and short range interaction, there is at most one invariant measure. 
It is interesting to remark the connection of the above with the problem of phase transition.Phase transition is linked to the presence of more than one DLR measure. It is conjectured that, as in the compact case [8], there is no phase transition in one dimension if asymptotically $\left|J_{x y}\right| \sim|x-y|^{-2-\varepsilon}$ in the sense that there is just one regular DLR measure. In some cases this has in fact been proven and our result shows that absence of phase transition can be extended to the class of invariant measures.

While the situation is quite clear in the one dimensional case it is completely open for higher dimensions. The argument which we present in this paper is essentially one dimensional. In higher dimension we could again prove that with probability one infinitely many spins are uniformly bounded, but what we really need is to prove that there are infinitely many closed contours going to infinity on which the spins are uniformly bounded and we were not able to show this conjecture.

In Sect. 2 we give a quite detailed proof of the result quoted above.

In Sect. 3 we shortly discuss the harmonic oscillator (Gaussian) case with nearest neighbor interactions and we "reduce" the problem to that of studying a 2-dimensional Markov process with deterministic transition probabilities.

\section{One Dimensional Case}

\section{The Model}

Theinfinite spin system is the measurable space $\mathscr{X}=\mathbb{R}^{\mathbb{Z}}$ with the $\sigma$-algebra of Borel sets inherited by the product topology in $\mathscr{X}$. We use the same notation as [2], therefore $\underline{S} \in \mathscr{X}, \underline{S}=\left(S_{x}, x \in \mathbb{Z}\right), S_{\Lambda}=\left(S_{x}, x \in \Lambda\right) \Lambda \subset \mathbb{Z}$. Our model is specified by a self-energy and a pair interaction. The energy of a configuration $S_{A}, \Lambda \subset \mathbb{Z}$ finite, is given by

$$
U\left(S_{\Lambda}\right)=\sum_{x \in A} F\left(S_{x}\right)-\sum_{\substack{\{x, y\} \in \Lambda \\ x \neq y}} J_{x y} S_{x} S_{y}
$$

where $F: \mathbb{R} \rightarrow \mathbb{R}$ continuously, the sum in Eq. (2.1) is over all the non ordered pairs in $\Lambda$ of distinct points and

$$
J_{x y}=J(|x-y|) \quad \sum_{i \in \mathbb{Z}}|J(|i|)|<+\infty
$$

We assume that the system is superstable, that is there are $A>0$ and $B$ such that for any finite $\Lambda$ and any $S_{\Lambda}$

$$
U\left(S_{\Lambda}\right) \geqq \sum_{x \in \Lambda}\left(A S_{x}^{2}-B\right)
$$

Eq. (2.3) holds if $F\left(S_{x}\right)$ diverges quadratically in $S_{x}$ as $\left|S_{x}\right| \rightarrow+\infty$ with sufficiently large coefficient. We finally assume that there is $M \in \mathbb{Z}^{+}$such that

$$
|J(i)| \text { is decreasing for } i \geqq M
$$

While the assumption that $F$ is continuous can easily be weakened, assumption Eq. (2.4) is technically important, and we will point out when it is really needed.

DLR equations are given via prescribed conditional probabilities, which define finite volume Gibbs measures with pure boundary conditions. For finite $\Lambda$ and fixed 
external configuration $S_{\Lambda^{c}}$ the Gibbs measure is:

$$
\begin{aligned}
& q\left(d S_{\Lambda} \mid S_{\Lambda^{c}}\right)=Z_{\Lambda}^{-1}\left(S_{\Lambda^{c}}\right) d S_{\Lambda} \exp \left\{-\beta\left(U\left(S_{\Lambda}\right)+W\left(S_{\Lambda}, S_{\Lambda^{c}}\right)\right)\right\} \\
& d S_{\Lambda}=\prod_{x \in \Lambda} d S_{x} \quad d S_{x}=\text { Lebesgue measure on } \mathbb{R} \\
& W\left(S_{\Lambda}, S_{\Lambda^{c}}\right)=-\sum_{x \in \Lambda} \sum_{y \notin \Lambda} J_{x y} S_{x} S_{y} \\
& Z_{\Lambda}^{-1}\left(S_{\Lambda^{c}}\right) \quad \text { is the normalization factor. }
\end{aligned}
$$

Of course the meaning of Eq. (2.7) has to be specified: we define $W\left(S_{\Lambda}, S_{\Lambda^{c}}\right)$ only for external configurations which are in $G(\Lambda)$

$$
S_{\Lambda^{c}} \in G(\Lambda) \quad \text { iff } \quad \sum_{y \notin \Lambda}\left|J_{x y} S_{y}\right|<+\infty \forall x \in \Lambda
$$

$G(\Lambda)$ is a Borel set.

It is convenient to consider the Gibbs measures as measures on $\mathscr{X}$ via the following $q\left(d \underline{S} \mid \bar{S}_{\Lambda^{c}}\right)=q\left(d S_{\Lambda} \mid \bar{S}_{\Lambda^{c}}\right) \prod_{x \notin A} \delta\left(S_{x}-\bar{S}_{x}\right) d S_{x}$

where $\delta\left(S_{x}-\bar{S}_{x}\right)$ is the delta function.

Then a DLR measure $v$ is a probability measure with support on $\bigcap_{\Lambda \text { finite }} G(\Lambda)$ and conditional probabilities given by $q\left(d \underline{S} \mid S_{\Lambda^{c}}\right)$. That is, given any bounded $\Lambda$ in $\mathbb{Z}$ and any $A v$-measurable set we have

$$
v\left(A \mid \pi_{\Lambda^{c}}(\underline{S})\right)=q\left(A \mid S_{\Lambda^{c}}\right) \quad v \text {-a.e. }
$$

where $\pi_{\Lambda^{c}}$ is the partition which specifies the spins $S_{x}$ for every $x \notin \Lambda$ and $\pi_{\Lambda^{c}}(\underline{S})$ is the atom of $\pi_{\Lambda^{c}}$ containing $\underline{S}$. This definition implies that the functions

$$
w_{x}(\underline{S})=\sum_{y \neq x}\left|J(|x-y|) S_{y}\right|
$$

are measurable and finite with $v$-probability one.

\section{Regular DLR Measures}

The DLR measure $v$ is regular if its densities $\varrho\left(S_{\Lambda}\right)$, defined as

$$
v\left(d S_{\Lambda}\right)=\varrho\left(S_{\Lambda}\right) d S_{\Lambda} \quad \Lambda \text { finite } \varrho \in L^{1}\left(\mathbb{R}^{\Lambda}, d S_{\Lambda}\right),
$$

satisfy the condition: there exist $\gamma>0$ and $\delta \in \mathbb{R}$ such that for each $\Lambda$ finite

$$
\varrho\left(S_{\Lambda}\right) \leqq \exp \left\{-\sum_{x \in \Lambda}\left(\gamma S_{x}^{2}-\delta\right)\right\}
$$

There are theorems which prove the existence of regular DLR measures and characterize them either by support properties or as limits of finite volume Gibbs measures [1-3]. It is proven that $\gamma$ and $\delta$ depend only on the interaction $\left(\beta, F, J_{x y}\right)$ and not on $v$ as soon as it is known that $v$ is regular(see Corollary 5.3 of [1] and Theorem 1.1 of [3]). 
Theorem 2.1. For the model previously introduced every translationally invariant $D L R$ measure $v$, is regular.

The proof is given in several steps.

Step I. There is a set $\mathscr{X}_{0} \subset \mathbb{R}^{\mathbb{Z}}$ such that $v\left(\mathscr{X}_{0}\right)=1$ and with the following property. Let $\underline{S} \in \mathscr{X}_{0}$, then there is $C(\underline{S})<+\infty$ and a divergent increasing sequence $x_{n} \in \mathbb{Z}^{+}, x_{n}>M$ such that

$$
w_{x_{n}-M}(\underline{S}) \leqq C(\underline{S}), \ldots, w_{x_{n}}(\underline{S}) \leqq C(\underline{S}) ; \quad w_{-x_{n}}(\underline{S}) \leqq C(\underline{S}), \ldots, w_{-x_{n}+M}(\underline{S}) \leqq C(\underline{S})
$$

where $w_{x}(\underline{S})$ was introduced in Eq. (2.10), and $M$ in Eq. (2.4).

Proof. By definition $w_{x}(\underline{S})=\infty$ in a set of $v$ measure zero, therefore there is an increasing unbounded function $\varphi: \mathbb{R}^{+} \rightarrow \mathbb{R}^{+}$such that:

$$
\varphi\left[w_{0}(\underline{S})\right] \in L^{1}\left(\mathbb{R}^{\mathbb{Z}}, v\right) .
$$

Since $v$ is translationally invariant we can use Birkhoff theorem to define $(v$-a.e. $)$ the $L^{1}\left(\mathbb{R}^{\mathbb{Z}}, v\right)$-function

$$
\bar{\varphi}(\underline{S})=\lim _{n \rightarrow \infty} \frac{1}{\left|\Lambda_{n}\right|} \sum_{x \in \Lambda_{n}} \varphi\left[w_{x}(\underline{S})\right]
$$

where $\Lambda_{n}=(-n, \ldots, n) \subset \mathbb{Z}$. Let

$$
\Gamma(\underline{S}, d)=\left\{x \in \mathbb{Z}:|x|>M, w_{|x|-i}(\underline{S}) \leqq d, w_{-|x|+i}(\underline{S}) \leqq d, i=0, \ldots, M\right\}
$$

then if $|\Gamma(\underline{S}, d)|$ is finite, $\bar{\varphi}(\underline{S})>\varphi(d) /(2 M+2)$. This is easily seen by the following argument:

$$
\begin{aligned}
& \bar{\varphi}(\underline{S})= \lim _{n \rightarrow \infty}\left\{\frac{1}{\left|\Lambda_{n}\right|} \sum_{x \in \Lambda_{n} \backslash \Gamma} \varphi\left[w_{x}(\underline{S})\right]\right. \\
&\left.+\frac{1}{\left|\Lambda_{n}\right|} \sum_{x \in \Lambda_{n} \cap \Gamma} \varphi\left[w_{x}(\underline{S})\right]\right\} \\
& \geqq \lim _{n \rightarrow \infty} \frac{1}{\left|\Lambda_{n}\right|}\left(\left|\Lambda_{n}\right|-|\Gamma|\right) \frac{\varphi(d)}{2 M+2}=\frac{\varphi(d)}{2 M+2} \quad \Gamma \equiv \Gamma(\underline{S}, d) .
\end{aligned}
$$

This proves the theorem for the set $\mathscr{X}_{0}=\{\underline{S}: \bar{\varphi}(\underline{S}) \neq+\infty\}$ and for

$$
C(\underline{S})>\varphi^{-1}(4 M \bar{\varphi}(\underline{S}))
$$

which is well defined because $\varphi$ is increasing and unbounded.

Step II. Fix $\Delta \subset \Lambda$ finite in $\mathbb{Z}$ and define $\varrho_{\Lambda}\left(d S_{\Delta} ; S_{\Lambda^{c}}\right)$ as the density w.r.t. $d S_{\Delta}$ of the measure $q\left(d S_{\Lambda} / S_{A^{c}}\right)$ restricted to $\left\{S_{\Delta}\right\}$. For $\tilde{S} \in \mathscr{X}_{0}$ let $\left\{x_{n}\right\}$ and $C(\underline{\tilde{S}})$ be as in Step I, then there are $\tilde{\gamma}>0, \tilde{\delta}$ depending possibly on $\underline{\underline{S}}$, such that

$$
\begin{aligned}
& \left(\Lambda_{x_{n}}=\left(-x_{n}, \ldots, x_{n}\right)\right) \\
& \varrho_{\Lambda_{x_{n}}}\left(S_{\Delta} ; \tilde{S}_{\Lambda_{x_{n}}}\right) \leqq \exp \left[-\sum_{x \in \Delta}\left(\tilde{\gamma} S_{x}^{2}-\tilde{\delta}\right)\right]
\end{aligned}
$$

for any sufficiently large $n$ so that $\Lambda_{x_{n}} \supset \Delta$. 
Proof. This is a straightforward consequence of Ruelle's superstability estimates. In fact

$$
\begin{aligned}
U\left(S_{\Lambda}\right)+W\left(S_{\Lambda}, \tilde{S}_{\Lambda^{c}}\right) & =\sum_{x \in \Lambda}\left[F\left(S_{x}\right)-h_{x, \Lambda} S_{x}\right]-\sum_{\substack{\{x, y\} \in \Lambda^{\cdot} \\
x \neq y}} J_{x y} S_{x} S_{y} \\
h_{x, \Lambda} & =\sum_{y \notin \Lambda} J_{x y} \tilde{S}_{y} \quad x \in \Lambda
\end{aligned}
$$

so that the presence of external spins determines a new (non-invariant) magnetic field $h_{x, \Lambda}$ and a Gibbs measure with zero boundary conditions. We can apply superstability theorems $[1,2]$ because for any $\Lambda_{x_{n}}$ the magnetic field is uniformly estimated in terms of $C(\underline{\tilde{S}})$ and the thesis is obtained. Here an essential role is played by the condition expressed by Eq. (2.4); non-monotonicity can be controlled only for finitely many sites [M] via Eq. (2.16). We need to assume that if $h_{x, A}$ is bounded for $x M$-close to the boundaries then it is bounded by the same constant for all $x$ in $\Lambda$ and this is just ensured by Eq. (2.4). We note that the superstability theorem requires the assumption that the interaction is lower regular. This condition is weaker than Eq. (2.4).

Step III. Let $\pi_{\infty}$ be the $v$-measurable partition at infinity of $\mathbb{R}^{\mathbb{Z}}$, denote by $\{a\}$ the atoms of $\pi_{\infty}: \pi_{\infty}=\{a\}$, then the conditional probabilities of $\nu$ w.r.t. $\pi_{\infty}$ are denoted by $v(d \underline{S} \mid a)$. They havesupport on a and stillare DLR measures(for $v$-almostall $a$ ). For any $v$-measurable set $A$ we have

$$
v(A)=\int v(d \underline{S}) v(A \mid a(\underline{S}))
$$

where $a(\underline{S})$ is the atom of $\pi_{\infty}$ containing $\underline{S}$. Further for any $v$-measurable set $A$ we have that

$$
\lim _{n \rightarrow+\infty} q\left(A \mid S_{\Lambda_{n}^{c}}\right)=v(A \mid a(\underline{S})) \quad v \text {-a.e. }
$$

Proof. The definition of measurable partitions can be found in [9]. That $v(d \underline{S} \mid a)$ are $v$-a.e. DLR measures is a consequence of a well known property of conditional probabilities w.r.t. ordered partitions, [10]: the partition at infinity is coarser than the partition $\pi_{A^{c}}$ which specifies the spins outside of $\Lambda$. Equation (2.22) is just Doob's theorem, [11], for decreasing partitions because $v$ is DLR and therefore $q\left(A \mid S_{\Lambda_{n}^{c}}\right)$ $=v\left(A \mid \pi_{\Lambda_{n}^{c}}(\underline{S})\right)$ where $\pi_{\Lambda_{n}^{c}}(\underline{S})$ is the atom of $\pi_{\Lambda_{n}^{c}}$ which contains $\underline{S}$.

Step IV. There is a set $\mathscr{X}_{0}^{\prime} \subset \mathscr{X}_{0}, v\left(\mathscr{X}_{0}^{\prime}\right)=1$ such that the following holds. Define for $m, N \in \mathbb{Z}^{+}$

$$
\begin{aligned}
G_{m, N} & =\left\{\underline{S} \in \mathscr{X}: \sum_{x \in \Lambda_{m}} S_{x}^{2} \leqq N^{2}\left|\Lambda_{m}\right|\right\} \\
G & =\bigcup_{N} \bigcap_{m} G_{m, N}
\end{aligned}
$$

then for any $\underline{S} \in \mathscr{X}_{0}^{\prime}, v(G \mid a(\underline{S}))=1$.

Proof. The sets $G_{m, N}$ are countably many, therefore the set $\mathscr{X}_{0}^{\prime} \subset \mathscr{X}_{0}$ can be chosen so that Eq. (2.22) holds for $\underline{S} \in \mathscr{X}_{0}^{\prime}, A=G_{m, N}^{c}$ (complement of $\left.G_{m, N}\right)$ and $v(d \underline{S} \mid a(\underline{S}))$ is DLR. 
An estimate for $v\left(G_{m, N} \mid a(\underline{S})\right)$ can be obtained via Eq. (2.22) by considering the sequence $\Lambda_{x_{n}}$ and Eq. (2.18). Namely:

$$
\begin{aligned}
v\left(G_{m, N}^{c} \mid a(\underline{S})\right) & \leqq \int d S_{\Lambda_{m}} \chi_{G_{m}^{c}, N}(\underline{S}) \exp \left\{-\sum_{x \in \Lambda_{m}}\left(\tilde{\gamma} S_{x}^{2}-\tilde{\delta}\right)\right\} \\
& \leqq \exp \left\{-\left(\frac{\tilde{\gamma}}{2} N^{2}-\bar{\delta}\right)\left|\Lambda_{m}\right|\right\}
\end{aligned}
$$

where $\bar{\delta}=\tilde{\delta}+\lg \sqrt{\frac{\pi}{\gamma / 2}}$. Hence

$$
\begin{aligned}
v\left(\bigcap_{m} G_{m, N} \mid a(\underline{S})\right) & \geqq 1-\sum_{m} v\left(G_{m, N}^{c} \mid a(\underline{S})\right) \\
& \geqq 1-\sum_{m}\left(e^{-\left(\gamma / 2 N^{2}-\bar{\delta}\right)}\right)^{2 m+1} \underset{N \rightarrow+\infty}{\longrightarrow} 1
\end{aligned}
$$

Step $V$. For $\underline{S} \in \mathscr{X}_{0}^{\prime}, v(d \underline{S} \mid a(\underline{S}))$ is regular and as a consequence also $v$ is regular.

Proof. $v(d \underline{S} \mid a(\underline{S}))$ is regular because of a superstability theorem which shows the equivalence between the regularity condition here given and the support property shown in Step IV, (see Corollary 5.3 of [1] and Theorem 4.4 of [3]). By the observation noted in the definition of regular measures, Eq. (2.12) holds for $\gamma$ and $\delta$ not depending on $a(\underline{S})$, when $\underline{S} \in \mathscr{X}_{0}^{\prime}$, therefore Eq. (2.21) proves that also $v$ is regular.

By this Theorem 2.1 has been proven.

\section{Concluding Remarks}

There are several conceivable extensions of the results obtained in this paper. One could easily allow interactions which differ from ours by bounded potentials. It would also seem possible to treat many-body interactions (e.g. $J_{x y z} S_{x}^{\alpha} S_{y}^{\beta} S_{z}^{\gamma}, \alpha, \beta, \gamma$ positive integers) as far as Ruelle's superstability estimates hold (in the sense of Corollary 2.3 of [2]). Also some monotonicity condition as in Eq. (2.4) should have to be imposed. However the most interesting extension is, in our opinion, the generalization to several dimensions. This is quite a hard problem as indicated by the following example.

Consider the two dimensional model in $\mathbb{R}^{\mathbb{Z}^{2}}$ given by the following conditional probabilities :

$$
q\left(d u_{x} \mid u_{\{x\} \mathrm{c}}\right)=\delta\left(u_{x}-\lambda \sum_{\langle x, y\rangle} u_{y}\right) d u_{x}
$$

where $\langle x, y\rangle$ means nearest neighborhood (in $\mathbb{Z}^{2}$ ), $\delta$ is the delta function and $\lambda<\frac{1}{4}$.

Since it is easy to prove that $\prod_{x} \delta\left(u_{x}\right) d u_{x}$ is the only invariant measure such that $\int Q(d u)\left|u_{x}\right|<+\infty$, the conjecture amounts to the following question : are there any translationally invariant probability measure $Q(d u)$ with conditional probabilities given by Eq. (3.1) other than $\prod_{x} \delta\left(u_{x}\right) d u_{x}$ ?

We were not able to give an answer in the general case. We mention that it can be proven (along thelines of what has been donein [6]) that all the DLR measures $v$ for the Gaussian 2-dimensional case can be written as

$$
v(d \underline{S})=\int_{u \in \mathbb{R}^{\mathbb{Z}^{2}}} Q_{v}(d u) v_{0}(d(\underline{S}-\underline{u}))
$$


where $Q_{v}$ is a solution of the DLR equation with conditional probabilities given by Eq. (3.1), $v_{0}$ is the only regular DLR measure for the Gaussian case, [4], characterized by

$$
\begin{aligned}
J_{x y} & = \begin{cases}J & \text { if } x \text { and } y \text { are nearest neighborhood } \\
0 & \text { otherwise }\end{cases} \\
F\left(S_{x}\right) & =k S_{x}^{2}
\end{aligned}
$$

and

$$
\lambda=J / 2 k
$$

Acknowledgments. I am deeply indebted to G. Benfatto, G. Gallavotti and E. Presutti for many helpful discussions.

\section{References}

1. Ruelle, D.: Superstable interactions in classical statistical mechanics. Commun. Math. Phys. 18, 127-159 (1970)

2. Ruelle, D.: Probability estimates for continuous spin systems. Commun. Math. Phys. 50, 189-194 (1976)

3. Lebowitz, J.L., Presutti, E.: Statistical mechanics of systems of unbounded spins. Commun. Math. Phys. 50, 195-218 (1976)

4. Cassandro, M., Olivieri, E., Pellegrinotti, A., Presutti, E.: Existence and uniqueness of DLR measures for unbounded spin systems. Z. Wahrscheinlichkeitstheorie verw. Gebiete 41, 313-334 (1978)

5. Pirlot, M.: A strong variational principle for continuous spin systems. Preprint (1978)

6. Benfatto, G., Presutti, E., Pulvirenti, M.: DLR measures for one dimensional harmonic systems. Z. Wahrscheinlichkeitstheorie verw. Gebiete 41, 305-312 (1978

7. Kesten, H.: Existence and uniqueness of countable one-dimensional Markov random fields. Ann. Prob. 4, 557-569 (1976)

8. Gallavotti, G., Miracle-Sole, S. : Absence of phase transitions in hard-core one-dimensional systems with long range interactions. J. Math. Phys. 11, 147-154 (1969)

Dobrushin, R.L.: Gibbsian random fields for lattice systems with pair interactions. Funkt. Anal. Ego Pril. 2, 31-43 (1968)

Dobrushin, R.L.: Problem of uniqueness of the Gibbsian random field and the problem of phase transitions Funkt. Anal. Ego Pril. 2, 44-57 (1968)

9. Rohlin, V.A.: On the fundamental ideas of measure theory. Am. Math. Soc. Transl. 10, 1-54 (1962)

10. Preston, C.: Random fields. Lectures notes in mathematics, Vol. 534. Berlin, Heidelberg, New York: Springer 1976

11. Neveu, J.: Mathematical foundations of the calculus of probability. San Francisco, Calif. : Holden Day 1965

Communicated by E. Lieb

Received June 30, 1978; in revised from November 15, 1978 